\title{
Stability and invariant manifold for a predator-prey model with Allee effect
}

\author{
Ünal Ufuktepe ${ }^{1 *}$, Sinan Kapçak and Olcay Akman²
}

\section{"Correspondence:}

unal.ufuktepe@ieu.edu.tr

'Department of Mathematics, Izmir

University of Economics, İzmir,

Turkey

Full list of author information is

available at the end of the article

\begin{abstract}
Predator-prey models are similar to host-parasite and host-parasitoid models. We investigate the stability and invariant manifolds of a discrete predator-prey model by using center manifold theory which is not addressed in Çelik and Duman (Chaos Solitons Fractals 40:1956-1962, 2009), and Wang et al. (Ecol. Complex. 8:81-85, 2011).
\end{abstract}

\section{Introduction}

Predator-prey models are similar to host-parasite and host-parasitoid models. In this system, the predator does not live on the host. The prey serves as a food source for the predator. There are many interactions between any pair of biological species; if two species compete for limited resources or one of the species preys upon the other, then one of the species is extinct.

The dynamic relationship between the interacting species has been one of the dominant themes in both ecology and mathematical ecology due to its universal importance. Recently many authors have explained the dynamics of competition and predator-prey models.

The following discrete time prey-predator system was studied by Çelik and Duman with an Allee effect on the prey population [1], and by Wang, Zhang, and Liu with Allee effects both on prey and predator [2]:

$$
\begin{aligned}
& N_{t+1}=N_{t}+r N_{t}\left(1-N_{t}\right)-a N_{t} P_{t}, \\
& P_{t+1}=P_{t}+a P_{t}\left(N_{t}-P_{t}\right),
\end{aligned}
$$

where the parameters $a, r$ are positive, $N_{t}$ is prey density at time $t$ and $P_{t}$ is predator density at time $t, r$ is the intrinsic growth rate. The term $a N_{t}$ is per capita predator increase due to prey consumption. Çelik and Duman [1] claimed that the eigenvalues for the fixed point $(0,0)$, which is a non-hyperbolic fixed point, are $\lambda_{1}=1$ and $\lambda_{2}=1$, but the corresponding Jacobian matrix for this point is

$$
J_{0}=\left(\begin{array}{cc}
1+r & 0 \\
0 & 1
\end{array}\right)
$$

with the eigenvalues $\lambda_{1}=1+r$ and $\lambda_{2}=1$. Then $(0,0)$ is an unstable fixed point by the Jury condition [3].

(02013 Ufuktepe et al.; licensee Springer. This is an Open Access article distributed under the terms of the Creative Commons Attribution License (http://creativecommons.org/licenses/by/2.0), which permits unrestricted use, distribution, and reproduction in any medium, provided the original work is properly cited. 
The Allee effect was first described by ecologist Allee [4]. The Allee effect may be caused by a variety of mechanisms applicable in small populations. The presence of Allee effects indicates that there is a minimal population size necessary for a population to maintain itself in nature. Interest in the dynamics of small populations, including Allee effects, has increased in recent years. Interest in how small populations interact with other populations, including predator-prey systems, has also increased. Allee effects can occur at the higher tropic level (e.g., predator, parasite), at the lower level (e.g., prey, host), or during interaction between these levels. Adding Allee effects to a predator-prey system can be destabilizing, depending on the formulation of the equations and where Allee effects are added [5].

In this paper, we consider the system subject to equations (1) when the predator population is subject to an Allee effect which is more general than described by the system of equations (3.1) in [2], and we also use the center manifold theory for non-hyperbolic equilibrium points [3].

$$
\begin{aligned}
& N_{t+1}=N_{t}+r N_{t}\left(1-N_{t}\right)-a N_{t} P_{t}, \\
& P_{t+1}=P_{t}+a P_{t}\left(N_{t}-P_{t}\right) \frac{P_{t}^{d}}{m+P_{t}^{d}},
\end{aligned}
$$

where the parameters $a, r, m$ are positive and $d \geq 1$. When $d=1$, the system is the model with an Allee effect on the predator discussed by Wang, Zhang, and Liu [2]. The aim of this paper is to investigate stability of model (2) and analyze the stability of the exclusion fixed point, which is non-hyperbolic, for the particular cases when $d=1$ and $d=2$ by using the center manifold theory which is not addressed in $[1,2]$.

\section{Stability of system (2)}

In this section we investigate the stability condition for system (2).

The fixed points are $(0,0),(1,0)$, and $\left(\frac{r}{a+r}, \frac{r}{a+r}\right)$. The Jacobian matrix of the planar map in (2) is

$$
J=\left(\begin{array}{cc}
1+r-2 r N-a P & -a N \\
\frac{a P^{1+d}}{m+P^{d}} & \frac{m^{2}+(1+a(N-2 P)) P^{2 d}-m P^{d}(-2-a((1+d) N-(2+d) P))}{\left(m+P^{d}\right)^{2}}
\end{array}\right) .
$$

The Jacobian matrix for the extinction fixed point $(0,0)$ is

$$
J_{0}=\left(\begin{array}{cc}
1+r & 0 \\
0 & 1
\end{array}\right)
$$

$(0,0)$ is unstable since one of the eigenvalues $J_{0}$ is greater than 1 . The Jacobian for the exclusion fixed point $(1,0)$ is

$$
J_{1}=\left(\begin{array}{cc}
1-r & -a \\
0 & 1
\end{array}\right)
$$

Since one of the eigenvalues of the matrix $J_{1}$ is 1 , this point is non-hyperbolic. By using the center manifold theory in the next section, we will show that for some particular cases, this point is unstable. 


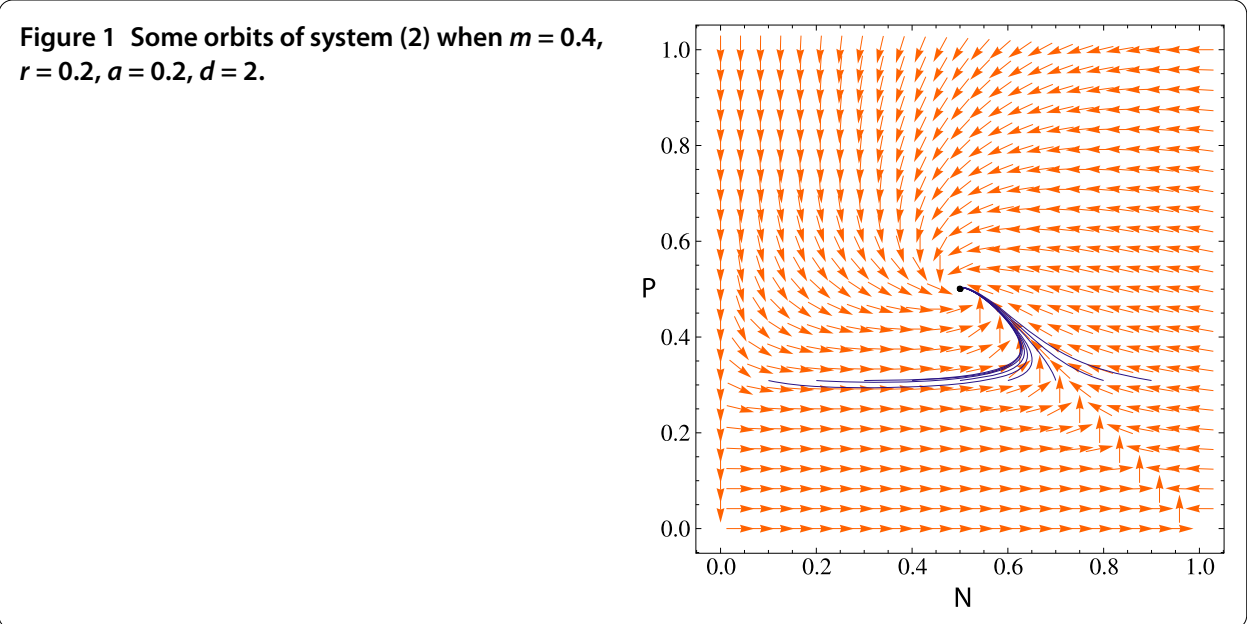

We now discuss the coexistence of the fixed point, i.e., $\left(\frac{r}{a+r}, \frac{r}{a+r}\right)$ : the Jacobian matrix for this point is

$$
J_{*}=\left(\begin{array}{cc}
\frac{a+r-r^{2}}{a+r} & -\frac{a r}{a+r} \\
\frac{a\left(\frac{r}{a+r}\right)^{1+d}}{m+\left(\frac{r}{a+r}\right)^{d}} & 1-\frac{a\left(\frac{r}{a+r}\right)^{1+d}}{m+\left(\frac{r}{a+r}\right)^{d}}
\end{array}\right) .
$$

By using the Jury conditions [3], after some manipulation, we obtain the following theorem.

Theorem 1 The positive fixed point $\left(\frac{r}{a+r}, \frac{r}{a+r}\right)$ is asymptotically stable if and only if

$$
0<r[(K+m) r+a(K-K r)]<4 a(K+m)-a K r-(K+m)(-4+r) r
$$

where $K=\left(\frac{r}{a+r}\right)^{d}$.

In Figure 1, we give the numerical evidence for some particular values of the parameters that the positive fixed point is asymptotically stable.

\section{Instability of an exclusion fixed point}

In this section, by using the center manifold theory, we show that for some values of $d$ for system (2), the fixed point $(1,0)$ is unstable.

Theorem 2 For system (2), the following statements hold true:

(a) If $d=1$, then $(1,0)$ is unstable.

(b) If $d=2$, then $(1,0)$ is unstable.

Proof The eigenvalues of the Jacobian matrix corresponding to the system given in (2) at the point $(1,0)$ are $\lambda_{1}=1-r$ and $\lambda_{2}=1$. If $r>2$, then $\left|\lambda_{1}\right|>1$ and $(1,0)$ is unstable.

Now, let us consider the case where $0<r<2$ in which the eigenvalues $\lambda_{1}, \lambda_{2}$ are subject to $\left|\lambda_{1}\right|<1$ and $\lambda_{2}=1$.

(a) In order to apply the center manifold theory, we make a change of variables in system (2) by shifting the point $(1,0)$ to $(0,0)$ with $u=N-1$ and $v=P$, for $d=1$, the new 
system is

$$
\begin{aligned}
& u_{t+1}=\left(u_{t}+1\right)-r\left(u_{t}+1\right) u_{t}-a\left(u_{t}+1\right) v_{t}-1, \\
& v_{t+1}=v_{t}+a v_{t}\left(u_{t}-v_{t}+1\right) \frac{v_{t}}{m+v_{t}} .
\end{aligned}
$$

The Jacobian of the planar map given in $(3)$ at $(0,0)$ is

$$
\tilde{J}_{0}=\left(\begin{array}{cc}
1-r & -a \\
0 & 1
\end{array}\right)
$$

According to system (3), it becomes

$$
\begin{aligned}
& u_{t+1}=(1-r) u_{t}-a v_{t}+\tilde{f}\left(u_{t}, v_{t}\right), \\
& v_{t+1}=v_{t}+\tilde{g}\left(u_{t}, v_{t}\right),
\end{aligned}
$$

where

$$
\tilde{f}\left(u_{t}, v_{t}\right)=-u_{t}\left(r u_{t}+a v_{t}\right)
$$

and

$$
\tilde{g}\left(u_{t}, v_{t}\right)=\frac{a\left(1+u_{t}-v_{t}\right) v_{t}^{2}}{m+v_{t}}
$$

Consider the center manifold $v=h(u)$. Let us assume that the map $h$ takes the form

$$
h(u)=-\frac{r}{a} u+\alpha u^{2}+\beta u^{3}+O\left(u^{4}\right), \quad \alpha, \beta \in \mathbb{R} .
$$

This leads to the evaluation of two constants $\alpha$ and $\beta$. The function $h$ must satisfy the center manifold equation

$$
h((1-r) u-a h(u)+\tilde{f}(u, h(u)))-h(u)-\tilde{g}(u, h(u))=0 .
$$

By using the Taylor series expansion, we solve the functional equation above yielding

$$
\alpha r-\frac{r^{2}}{m a}=0
$$

and

$$
\alpha r+\beta r+\frac{2 \alpha r}{m}-\frac{r^{2}}{m a}-\frac{r^{3}}{m^{2} a^{2}}-\frac{r^{3}}{m a^{2}}-2 \alpha\left(r+a\left(\alpha-\frac{r}{a}\right)\right)=0 .
$$

By solving the last set of equations, we obtain $\alpha=\frac{r}{m a}, \beta=\frac{r^{2}+m r^{2}}{m^{2} a^{2}}$. Hence

$$
h(u)=-\frac{r}{a} u+\frac{r}{m a} u^{2}+\frac{r^{2}+m r^{2}}{m^{2} a^{2}} u^{3} .
$$


Thus, on the center manifold $v=h(u)$, we have the following map:

$$
S(u)=-\frac{u\left((1+m) r^{2} u^{2}(1+u)+m a(-m+r u(1+u))\right)}{m^{2} a} .
$$

Since $S^{\prime}(0)=1$ and $S^{\prime \prime}(0)=-\frac{2 r}{m}<0$, the exclusion fixed point $(1,0)$ is unstable.

(b) Similarly, when $d=2$, the new system is given by

$$
\begin{aligned}
& u_{t+1}=\left(u_{t}+1\right)-r\left(u_{t}+1\right) u_{t}-a\left(u_{t}+1\right) v_{t}-1, \\
& v_{t+1}=v_{t}+a v_{t}\left(u_{t}-v_{t}+1\right) \frac{v_{t}^{2}}{m+v_{t}^{2}} .
\end{aligned}
$$

The Jacobian of the planar map which is given in $(5)$ at the point $(0,0)$ is

$$
\tilde{J}_{0}=\left(\begin{array}{cc}
1-r & -a \\
0 & 1
\end{array}\right) \text {. }
$$

The equations in system (5) become

$$
\begin{aligned}
& u_{t+1}=(1-r) u_{t}-a v_{t}+\tilde{f}\left(u_{t}, v_{t}\right), \\
& v_{t+1}=v_{t}+\tilde{g}\left(u_{t}, v_{t}\right),
\end{aligned}
$$

where

$$
\tilde{f}\left(u_{t}, v_{t}\right)=-u_{t}\left(r u_{t}+a v_{t}\right)
$$

and

$$
\tilde{g}\left(u_{t}, v_{t}\right)=\frac{a\left(1+u_{t}-v_{t}\right) v_{t}^{3}}{m+v_{t}^{2}} .
$$

Consider again the center manifold $v=h(u)$. Let us assume that the function $h$ takes the form

$$
h(u)=-\frac{r}{a} u+\alpha u^{2}+\beta u^{3}+O\left(u^{4}\right), \quad \alpha, \beta \in \mathbb{R} .
$$

Now we can evaluate the constants $\alpha$ and $\beta$. The function $h$ must satisfy the center manifold equation

$$
h((1-r) u-a h(u)+\tilde{f}(u, h(u)))-h(u)-\tilde{g}(u, h(u))=0 .
$$

Utilizing the Taylor expansion, we solve the above functional equation

$$
\alpha r=0
$$

and

$$
-2 a \alpha^{2}+(\alpha+\beta) r+\frac{r^{3}}{m a^{2}}=0 .
$$




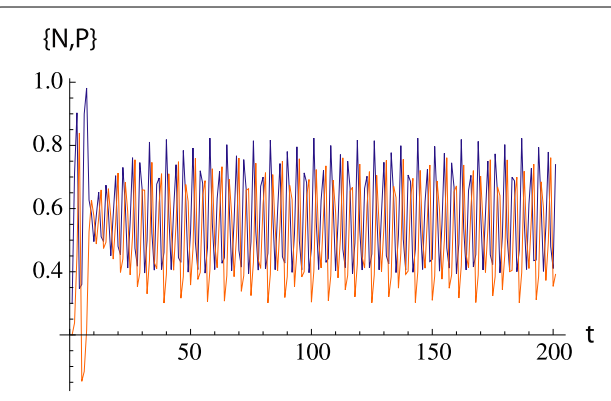

(a) without the Allee effect

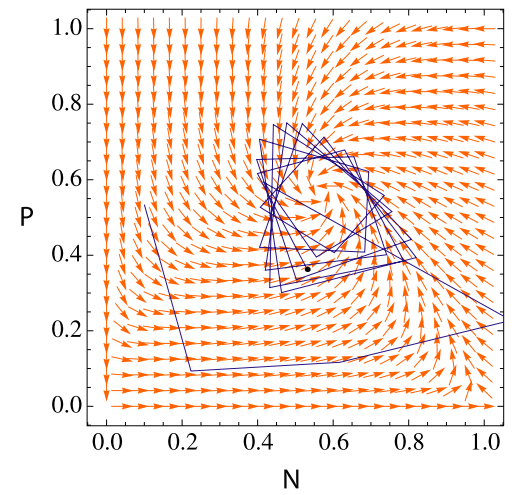

(c) without the Allee effect

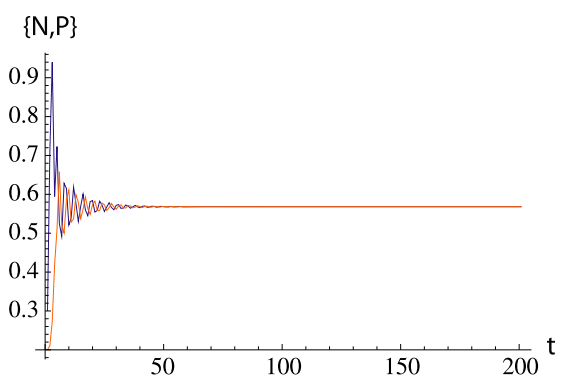

(b) with the Allee effect

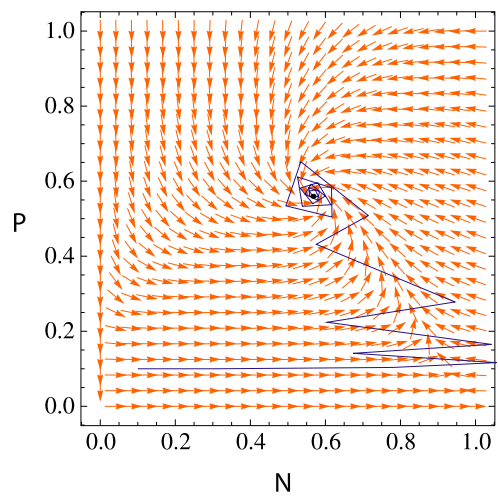

(d) with the Allee effect

Figure 2 Trajectories of the prey-predator system: the Allee effect stabilizes the system. $r=2.5, a=1.9$ $d=2$; (a), (c) $m=0$; (b), (d) $m=0.1$

Solution of the above set of equations is $\alpha=0, \beta=-\frac{r^{2}}{m a^{2}}$. Hence

$$
h(u)=-\frac{r}{a} u-\frac{r^{2}}{m a^{2}} u^{3} .
$$

Thus, on the center manifold $v=h(u)$, we have the following map:

$$
R(u)=\frac{m a u+r^{2} u^{3}(1+u)}{m a} .
$$

Since $R^{\prime}(0)=1$ and $R^{\prime \prime}(0)=0$, we need to calculate the Schwarzian derivative [3] at the origin. Since the Schwarzian derivative at the origin is $\frac{6 r^{2}}{m a}>0$, we conclude that the fixed point $(1,0)$ is unstable.

We show the influence of the Allee effect on the local stability of system (2). In Figures 2 and 3, we show the trajectories of predator-prey densities in the system we studied. Figure 2 shows that the corresponding equilibrium points can move from unstable to stable under the Allee effect. On the other hand, the Allee effect may be a destabilizing force in our predator-prey system which made the equilibrium point change from stable to unstable. Figure 3 depicts the fact. Table 1 also gives compact information about the stabilizing and destabilizing force of the Allee effect in our model. 


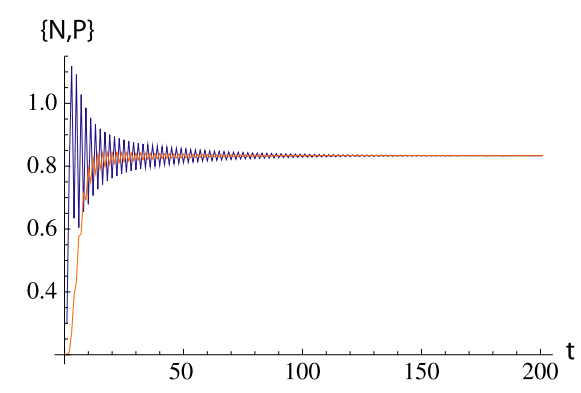

(a) without the Allee effect

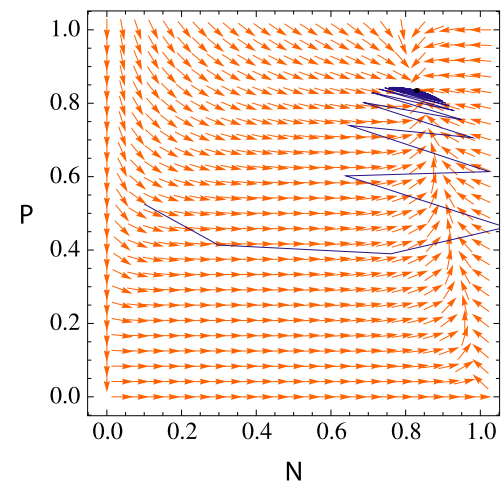

(c) without the Allee effect

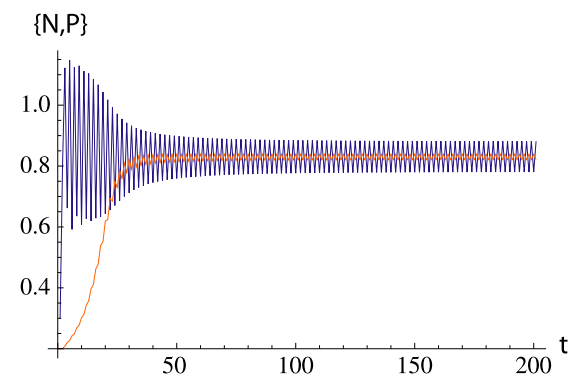

(b) with the Allee effect

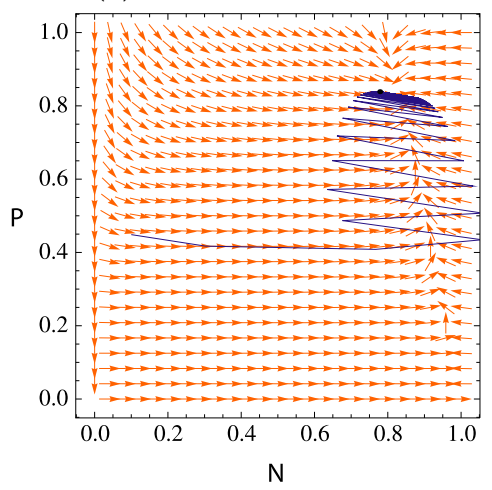

(d) with the Allee effect

Figure 3 Trajectories of the prey-predator system: the Allee effect destabilizes the system. $r=2.5$, $a=0.5, d=2 ;$ (a), (c) $m=0$; (b), (d) $m=0.3$.

Table 1 The Allee effect may stabilize or destabilize the system

\begin{tabular}{lllllll}
\hline Figure & $\boldsymbol{r}$ & $\boldsymbol{a}$ & $\boldsymbol{d}$ & $\boldsymbol{m}$ & Fixed point & Initial point \\
\hline 2(a), 2(c) & 2.5 & 1.9 & 2 & 0 & Unstable & $(0.3,0.2)$ \\
2(b), 2(d) & 2.5 & 1.9 & 2 & 0.1 & Stable & $(0.3,0.2)$ \\
3(a), 3(c) & 2.5 & 0.5 & 2 & 0 & Stable & $(0.3,0.2)$ \\
3(b), 3(d) & 2.5 & 0.5 & 2 & 0.3 & Unstable & $(0.3,0.2)$ \\
\hline
\end{tabular}

\section{Competing interests}

The authors declare that they have no competing interests.

\section{Authors' contributions}

All authors contributed equally and significantly in writing this paper. All authors read and approved the final manuscript.

\section{Author details}

${ }^{1}$ Department of Mathematics, İzmir University of Economics, İzmir, Turkey. ${ }^{2}$ Department of Mathematics, Illinois State University, Normal, USA.

Received: 11 April 2013 Accepted: 7 November 2013 Published: 03 Dec 2013

\section{References}

1. Çelik, C, Duman, O: Allee effect in a discrete-time predator-prey system. Chaos Solitons Fractals 40, 1956-1962 (2009)

2. Wang, W-X, Zhang, Y, Liu, C: Analysis of a discrete-time predator-prey system with Allee effect. Ecol. Complex. 8, 81-85 (2011)

3. Elaydi, S: Discrete Chaos: With Applications in Science and Engineering, 2nd edn. Chapman \& Hall/CRC, London (2008)

4. Allee, WC: The Social Life of Animals. Book Club, London (1941)

5. Jang, SRJ, Diamond, SL: A host-parasitoid interaction with Allee effects on the host. Comput. Math. Appl. 53, 89-103 (2007) 
10.1186/1687-1847-2013-348

Cite this article as: Ufuktepe et al.: Stability and invariant manifold for a predator-prey model with Allee effect. Advances in Difference Equations 2013, 2013:348

Submit your manuscript to a SpringerOpen ${ }^{\circ}$ journal and benefit from:

- Convenient online submission

- Rigorous peer review

- Immediate publication on acceptance

Open access: articles freely available online

- High visibility within the field

- Retaining the copyright to your article

Submit your next manuscript at $\gg$ springeropen.com 